\title{
Drawing enhances cross-modal memory plasticity in the human brain: a case study in a totally blind adult
}

\author{
Lora T. Likova* \\ The Smith-Kettlewell Eye Research Institute, San Francisco, CA, USA
}

\section{Edited by:}

Idan Segev, The Hebrew University of Jerusalem, Israel

Reviewed by:

David J. McGonigle,

Cardiff University, UK

Hidenao Fukuyama,

Kyoto University, Japan

*Correspondence:

Lora T. Likova, The Smith-Kettlewell

Eye Research Institute, 2318

Fillmore Street San Francisco,

CA 94115, USA.

e-mail: lora@ski.org
In a memory-guided drawing task under blindfolded conditions, we have recently used functional Magnetic Resonance Imaging (fMRI) to demonstrate that the primary visual cortex (V1) may operate as the visuo-spatial buffer, or "sketchpad," for working memory. The results implied, however, a modality-independent or amodal form of its operation. In the present study, to validate the role of V1 in non-visual memory, we eliminated not only the visual input but all levels of visual processing by replicating the paradigm in a congenitally blind individual. Our novel Cognitive-Kinesthetic method was used to train this totally blind subject to draw complex images guided solely by tactile memory. Control tasks of tactile exploration and memorization of the image to be drawn, and memory-free scribbling were also included. FMRI was run before training and after training. Remarkably, $\mathrm{V} 1$ of this congenitally blind individual, which before training exhibited noisy, immature, and non-specific responses, after training produced full-fledged response time-courses specific to the tactile-memory drawing task. The results reveal the operation of a rapid training-based plasticity mechanism that recruits the resources of $\mathrm{V} 1$ in the process of learning to draw. The learning paradigm allowed us to investigate for the first time the evolution of plastic re-assignment in $\mathrm{V} 1$ in a congenitally blind subject. These findings are consistent with a non-visual memory involvement of $\mathrm{V} 1$, and specifically imply that the observed cortical reorganization can be empowered by the process of learning to draw.

Keywords: drawing, blind, brain plasticity, primary visual cortex V1, working memory, visuo-spatial sketchpad, learning, fMRI

\begin{abstract}
“... we must look upon artists as persons whose observation of sensuous impression is particularly vivid and accurate, and whose memory for these images is particularly true."
\end{abstract}

Helmholtz, 1871

\section{INTRODUCTION}

We may not be aware of the complexity of drawing, but when analyzed in detail it becomes clear that drawing is an amazing process that requires precise orchestration of multiple brain mechanisms; perceptual processing, memory, precise motor planning and motor control, spatial transformations, emotions, and other diverse higher cognitive functions, are all involved. In terms of the multiple-intelligence theory (Gardner, 1983), drawing heavily employs such categories as bodily-kinesthetic and visuo-spatial intelligence.

This operational complexity may be one reason for the neglect of drawing as an experimental paradigm, being considered too complex to be successfully analyzed. In contrast to other arts, such as music, there have been only a few neuroimaging studies of the neural mechanisms of visual art, and of drawing in particular. In actuality, most of the available research on drawing (e.g., Makuuchi et al., 2003; Ferber et al., 2007; Ogawa and Inui, 2009) was heavily motivated by its importance in neurological tests, such as for the diagnosis of constructional apraxia
(Mayer-Gross, 1935; Piercy et al., 1960; de Renzi, 1982; Grossi and Trojano, 1999; Lee et al., 2004).

More than a century ago, in his famous 1871 lecture, Helmholtz pointed out that artists possess not only advanced observational capabilities, but also enhanced memory for the observed images. While the first part of this claim is often mentioned in vision science, the second-memory-related-part has been widely neglected, as though it did not reach the right audience.

We sought to understand if there is something especially advanced about artists' memory. And if so, is that advanced memory an inborn artistic trait or can it be engendered by the process of learning to draw? Drawing, and in particular memory-guided drawing, challenges the encoding of detailed spatial representations, their retrieval from memory and "projection" back onto a mental high-resolution "screen," so as to guide the motion of the drawing hand with the requisite precision.

\section{THE ROLE OF THE MEMORY BUFFER}

One theoretical construct that meets these demands is the visuospatial memory buffer also termed the "visuo-spatial sketchpad." In the classic model of working memory (as proposed by Baddeley and Hitch, 1974; Baddeley, 1986, 2000, 2003), this buffer is a major component that instantiates the function of developing and holding in working memory an accurate spatial representation of the retrieved object, providing a "sketch" that can be further 
spatially manipulated by the central executive to guide goaldirected behaviors. This influential model helps to understand the processes of memory encoding and retrieval for the kinds of spatial representations involved in the drawing task, allowing for the active maintenance of information about stimuli no longer in view. (It is not by chance that this landmark component of the real drawing process - the use of a disposable sketchpadwas the metaphor Baddeley employed for the memory module in question.) This model has provided major insights into functional neuroimaging of memory, and conversely, it has been successively updated based on neuroimaging data. For example, it has recently been proposed that working memory is not restricted to retention only, and that working memory and long-term memory may be functionally interrelated (e.g., Buchsbaum and D'Esposito, 2009; Ishai, 2009; Ranganath, 2009).

Where in the brain may the working memory "sketchpad" be implemented? Previous theoretical and neurophysiological studies in non-human primates (e.g., Mumford, 1991, 1996; Lee et al., 1998; Super et al., 2001a,b; Lee and Mumford, 2003; Super, 2003) had suggested that the primary visual cortex (area V1) may provide for the high-resolution visuo-spatial "sketchpad" function. These suggestions are based on the fact that V1 is unique in being the largest topographic map in the brain, with the highest spatial resolution, in addition to having a connectivity allowing parallel processing of the information from the whole map surface-these features being critically important for a successful "sketchpad" implementation.

Traditionally, however, all areas in the early visual cortex have been considered predominantly bottom-up, purely sensory, and devoted to the visual modality. Nevertheless, increasing evidence has shown that they are also subject to a number of top-down processes. Most recently, early visual cortex has been implicated in visual memory. It is now considered, for example, that this cortex, and V1 in particular, are not only important for processing information about the immediate sensory environment, but can also retain specific visual information for working memory over periods of many seconds in the absence of direct input to support higher-order cognitive functions (e.g., Williams et al., 2008; Harrison and Tong, 2009).

\section{THE AMODAL MEMORY HYPOTHESIS}

Furthermore, visual cortex can be activated in a number of nonvisual perceptual and memory tasks. It has been shown that verbal-memory can generate robust activation in the visual cortex of congenitally blind individuals (Amedi et al., 2003), and furthermore, episodic memory retrieval in congenitally blind individuals was associated with V1 activation (Raz et al., 2005).

The present study extends these results to memory in the tactile modality. We investigated whether V1 is involved in tactile working memory task in a congenitally blind individual. This question is of high importance for models of the functional architecture of human memory.

We have proposed that a highly demanding tactile-memory task, such as drawing guided solely by tactile-memory, is a powerful technique for addressing this question (Likova, 2010a, 2012). Beyond this, drawing has the unique advantage of providing an explicit readout of the memory content recalled during task performance, as it objectively "externalizes" the specific memory representation guiding the motor output in each trial.

Employing this novel memory paradigm in blindfolded subject, we have recently found that tactile-memory drawing strongly activates V1 (although no visual or even tactile information was available), while massively deactivating the entire extrastriate hierarchy (Likova, 2010a, 2012; Likova and Nicholas, 2010c). It is important to notice that this pattern of activation is quite distinct, almost the inverse of that for "classical" high-order functions, such as the known hierarchical pattern for visual imagery. The visual imagery signal propagates in top-down fashion through the visual hierarchy, being strongest in the higher extrastriate areas, decreasing towards the lower areas (e.g., Ishai and Sagi, 1995; Kreiman et al., 2000; O'Craven and Kanwisher, 2000; Kosslyn et al., 2001; Kosslyn and Thompson, 2003; Tong, 2003; Mechelli et al., 2004; Amedi et al., 2005; Merabet et al., 2005), and often not reaching V1 at all; thus, there is still an open debate whether imagery activates V1 itself or not. Notably, this visual imagery "signature" is entirely opposite to the occipital pattern generated by our drawing-from-tactile-memory task, which was characterized by having the strongest (and only) occipital activation in V1, while the extrastriate pathways were "cut off" by deactivation.

Consequently, the unique pattern of results in the blindfolded study was not compatible with an explicit role for visual imagery in this form of working memory. Instead, the strong V1 activation was more consistent with the hypothesis of the implementation of a working memory component, such as the spatial memory buffer, in this area. In view of the lack of any visual input under blindfolding, however, our findings suggest a re-conceptualization of the putative buffer as being modalityindependent or amodal.

In the current study, to further probe the amodal hypothesis, and to address the essential nature of drawing, we eliminated not only the visual input but any potential higher-level visual processing by selecting a congenitally blind novice. In contrast to late-onset blind individuals, who (similarly to the sighted) have had enough visual stimulation to develop vision and its associated visual imagery, visual memory, etc., congenitally blind individuals have had no access to visual information throughout life. Thus, congenital blindness, and even the wider category of early blindness, is considered to eliminate any visual influences of both bottom-up and top-down nature. In particular, it has been recognized as "clearly true that visual imagery does not account for cross-modal activation of visual cortex for the early blind" (Lacey et al., 2009), and that the congenitally blind are unable to perform visual imagery tasks (e.g., Goyal et al., 2006).

\section{DRAWING IN BLIND INDIVIDUALS}

Drawing, and visual art in general, is presumed to be highly dependent on the visual modality (as implicit in its specification as "visual art"). However, there are totally blind people, including those blind from birth, who have been able to develop visual art skills (e.g., Kennedy, 1993, 2000; Heller, 2000; Kennedy and Igor, 2003; Kennedy and Juricevic, 2006; Ponchillia, 2008). Perhaps the most famous congenitally blind artist is Esref Armagan from Turkey, who draws and even paints in color although he has 
never seen light. Astonishingly, this blind artist is able even to draw in one-point perspective, showing a respectable grasp of how horizontal lines converge to a point in the distance. Amedi et al. (2008) studied this artist using fMRI, and found activation in a widely distributed network, including not only frontal and parietal regions, but also brain areas normally associated with early vision, such as the calcarine sulcus. This is the only neuroimaging study of drawing in the blind prior to our studies. However, as Armagan was a professional painter with many decades of experience, these authors did not have the opportunity to study the process of learning-based brain reorganization itself, but only to look at the completed learning state. To our knowledge, therefore, our studies are the first on learning to draw in the blind, and on the corresponding dynamics of brain reorganization.

\section{THE NEED FOR THE COGNITIVE-KINESTHETIC TRAINING METHOD}

Our philosophy is that, because drawing encompasses a large range of demanding perception-to-action components, it provides for elaborated training in active spatial cognition. It forces the learners not just passively to explore the stimuli, but to develop detailed and stable memory representations in order to be able to re-express these representations in an explicit sensory format (i.e., to communicate the memory contents through drawing). These characteristics make drawing a potent paradigm for the study of memory.

Looking at line drawings by Matisse (e.g., Figure 1), we see how expressive only a few lines can be! Their appreciation seems such an effortless process that we are not aware of the invisible work of powerful brain mechanisms that provide the artist with the ability to transform $3 \mathrm{D}$ objects into their $2 \mathrm{D}$ projections by abstracting just the right contours into a line drawing; neither are we aware of how complex is the "inverse transformation" of such $2 \mathrm{D}$ drawings into an immediate understanding of the $3 \mathrm{D}$ objects that they represent.

It came as a surprise, therefore, to find that, when exposed for the first time to $2 \mathrm{D}$ raised-line drawings, many blind people have tremendous difficulty even in tactile recognition and comprehension of the 3D objects depicted. This negative finding, however, provided the opportunity to employ a learning paradigm in adults to investigate the developmental evolution of cognitive components of key importance for drawing, such as spatial memory.

In addition to the fact that the blind individuals are used to the haptic exploration of $3 \mathrm{D}$ shapes rather than their $2 \mathrm{D}$ projections

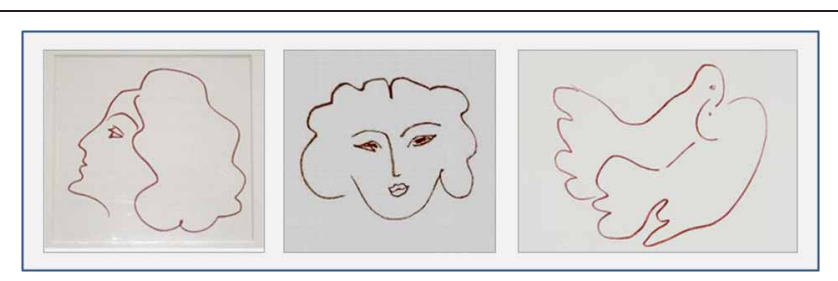

FIGURE 1 | Matisse: Lithographs No. 54: "Marie" (left), No. 35: "Ma Maîtresse" (center), No. 45: "Les Colombes Amoureuses" (right), from the illustrations for Ronsard's "Florilège des Amours" (1948). or abstract form, the explored 2D images are usually much larger than the pad of the index finger, so "scanning" movements of the finger along with the whole hand are needed to sense the entire image (Loomis and Klatzky, 2008). This requires an extensive spatiotemporal binding and memory of the continuously upgrading image. Thus, although there are some professional blind artists, both recognition of drawings and reproduction by drawing are extremely challenging for blind people. Furthermore, specific psychological barriers have to be faced and overcome, because most blind people find it difficult to believe that they would be able to learn to draw and would not even make the attempt.

All these considerations have been serious obstacles to conducting non-visual drawing studies, and have motivated the development of the Cognitive-Kinesthetic Method (Likova, $2010 a, b)$, which has proven to be both effective and inspirational for blind people. Key components of this learning method are the incorporation of top-down feedback through conceptual and/or spatial interpretations, and encouraging enjoyment from the learning process.

The congenitally blind individual of this study was welladapted to operating in the everyday spatial world, including longstanding familiarity with complex tactile manipulations and Braille reading, but had no writing or drawing experience. Functional Magnetic Resonance Imaging (fMRI) was run before and after the Cognitive-Kinesthetic training in order to investigate the dynamics of brain reorganization as a function of learning to draw. Although this congenitally blind individual was a mature adult, her brain showed dramatic functional reorganization. Most remarkably, V1, which exhibited no specific involvement before training, was massively recruited in the drawing task after training. Temporal waveform analysis revealed characteristic phases in the progression of the reorganization process.

\section{MATERIALS AND METHODS AN INNOVATIVE EXPERIMENTAL PLATFORM}

As there are no preceding neuroimaging studies of the kind, to make these studies possible it was necessary to develop a unique conceptual and experimental platform integrating a number of innovations, such as: (1) the Cognitive-Kinesthetic Method to effectively train people to draw without vision, (2) the first multisensory MRI-Compatible Drawing Tablet (for both tactile and visual drawing), incorporating a motion-capture system, (3) the first Method for estimating Topographic Maps in the Blind, (4) as well as implementation of standard probabilistic maps in blind individuals. This platform opens up a whole dimension of multimodal sensorimotor processing to neuroimaging studies.

\section{SUBJECT AND TRAINING}

The congenitally blind subject CB4 was a 61-year-old righthanded female, totally blind with no light perception, who lost her vision as a result of German measles (rubella) in her expectant mother, severely and permanently damaging the fetal optic nerves. The subject gave informed consent for the experimental protocol approved by the local research ethics committee, Institutional Review Board.

CB4 had not been previously studied by fMRI or behavioral methods of any kind. She is a sophisticated intellect and a fluent 
Braille reader, with a high education and lifetime employment, and was highly motivated to participate in the study. Nevertheless, despite her Braille fluency and longstanding familiarity with complex tactile manipulations, she had no experience with writing or drawing. Consequently, her training to draw had to start with the basics, such as the proper holding of the pen and key spatial concepts of the representation of $3 \mathrm{D}$ structure on a $2 \mathrm{D}$ plane. CB4 had relied heavily on active tactile exploration for her whole life, so it was quite surprising that she did not have a clear idea of elementary geometric concepts such as a straight line vs. a curve, right angles, etc., and was unable to reproduce any simple component through drawing. These issues were manifested at all levels of the experimental process - the tactile recognition and memorization phase, the memory recall in the drawing phase, the understanding of spatial relationships, and even the kinesthetic feedback and self-evaluation of her own performance. For example, she could think she had just drawn a straight line, while she actually drew an almost closed curve, and so on.

It became clear, however, that these "negatives" could be turned into significant "positives" that would for the first time allow tracking of the full evolution of the neural process of learning to draw. Another advantage was the fact that CB4 was an intelligent adult, able both to readily follow instructions and to express back her introspections.

Interestingly, it seems typical for blind people to expect that they would be unable to perform a task such as drawing without guidance from the non-drawing hand. Even the exceptional blind artist Armagan, despite his many decades of blind drawing, still used a technique that involved "holding the pencil in his right hand to draw, while following the created indentations with his left hand" (Amedi et al., 2008). The same study reported that he "cannot complete his drawings if he is not allowed to use his left hand to follow the indentations created by the drawing," meaning that he relied on tactile perception from the left hand to provide the configural feedback.

In contrast, the unique technique by which we trained CB4 taught her to draw without using any tactile feedback from the non-drawing (left) hand, thus focusing the training on the development of an effective memory representation to guide the drawing trajectory.

The training was performed for $1-1.5 \mathrm{~h}$ per day for five days during the week following the initial fMRI session. Our novel drawing method was able to inspire and to motivate CB4 to acquire the exciting drawing skill. Remarkably, after only a week of training, she advanced significantly relative to her starting level, although her capability was still not satisfactory to her. Two months later she came back for two "refresher" training sessions which she felt brought her up to an adequate skill level. To study the dynamics of the learning process, we ran fMRI before training, as well as after the prolonged period of consolidation and a refresher training session.

\section{EXPERIMENTAL DESIGN}

We used a three-task block paradigm, with interleaved baseline conditions (Figure 2). A battery of raised-line models of faces and objects was developed as the drawing targets (Figure 3). The three tasks were as follows: Explore/Memorize, E/M-perceptual

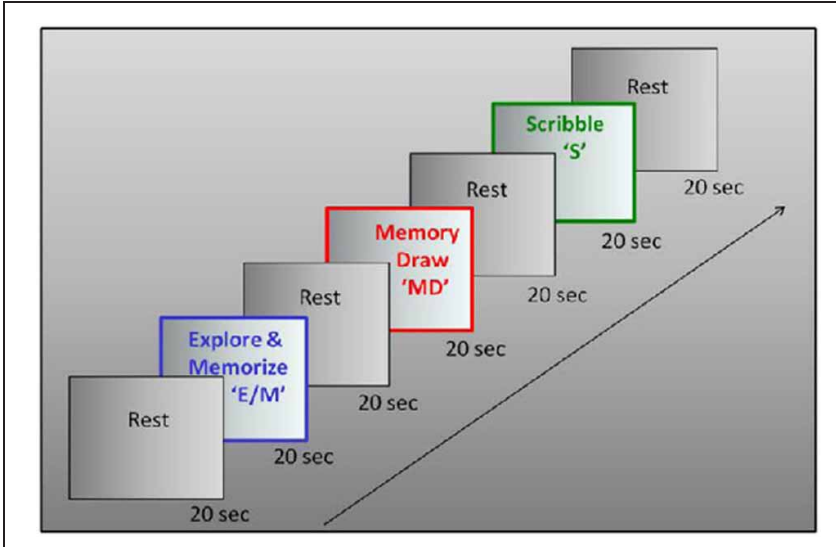

FIGURE 2 | Experimental design. Drawing was investigated in a three-phase paradigm consisting of a memory-guided drawing task, abbreviated as "MemoryDraw" (MD), plus two control tasks: a motor and "negative" memory control task "Scribble" (S), and a task of perceptual exploration and memorization of the model to be drawn

"Explore/Memorize" (E/M). Each task duration was 20 s, with 20 s rest intervals elapsing between the tasks, with the whole trial sequence being repeated 12 times in each scanning session.

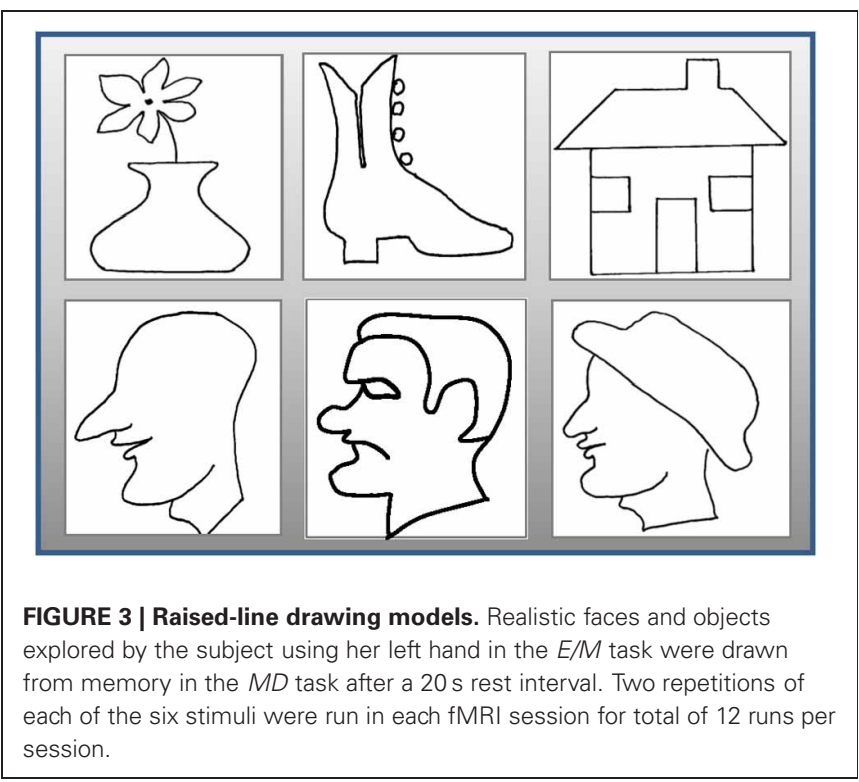

exploration and memorization of the model to be drawn; MemoryDraw, $M D$ — a memory-guided non-visual drawing task; and Scribble, $S$ - a motor-control and negative memory-control task. Each task duration was 20 s, with a 20 s baseline condition ("RestInterval," RI) intervening between the tasks during which the subject rested motionless being instructed to clear any image from mind. The start of each task or rest interval was prompted by an auditory cue. The whole three-task sequence with interleaved rest intervals ( $R I, E / M, R I, M D, R I, S)$ was repeated 12 times in each fMRI session.

One of the advanced aspects of the experimental design was that the models were always explored with the left hand but drawn by the right hand, thus requiring the subjects to develop 
a clear mental representation in order to transfer the information to the opposite (drawing) hand. This design ensures that in the MemoryDraw task the right (drawing) hand does not have any "haptic knowledge" of the image. Moreover, the fact that the left hand was not allowed to follow the contour drawn by the right hand ensures that the subject learns to draw without relying on any tactile configural feedback. Together, these design features enforce the encoding of a robust memory representation needed to guide the drawing trajectory.

In Explore/Memorize, using the left hand only, the subject had to tactually explore a raised-line drawing model on the left slot of the drawing tablet, and to develop a full memory representation of the image in preparation for the MemoryDraw task. Then the model image was removed, and the subject rested motionless for $20 \mathrm{~s}$ with no image in mind (RestInterval), followed by the MemoryDraw phase. In the following MemoryDraw phase the fiber-optic stylus was used to draw the image (from tactile memory) on the right slot of the tablet with the right hand. Scribble was a control for both the generic hand movement and memory involvement; the subject had to move the stylus with the right hand in a random trajectory over the right slot of the tablet to the extent and rate similar to the drawing movements, but under instructions not to plan or imagine any particular trajectory form, avoiding any cognitive content.

\section{TACTILE STIMULUS PRESENTATION AND HAND MOVEMENT CONTROL Custom-built, multisensory MRI-compatible drawing system}

To run drawing studies in the scanner is not a conventional protocol and faced a lot of unresolved technological problems. We developed a special-purpose drawing system that: (1) is MRIcompatible, (2) is ergonomically adaptable, (3) allows multiple tactile images to be presented in the scanner, (4) captures and records the drawing trajectory with high precision, and (5) provides a real-time visual feedback when drawing in the sighted is studied. This system (Figure 4) incorporates a dual-slot drawing tablet that is height/distance adjustable and an adapted version of a fiber-optic device for motion-capture of the drawing

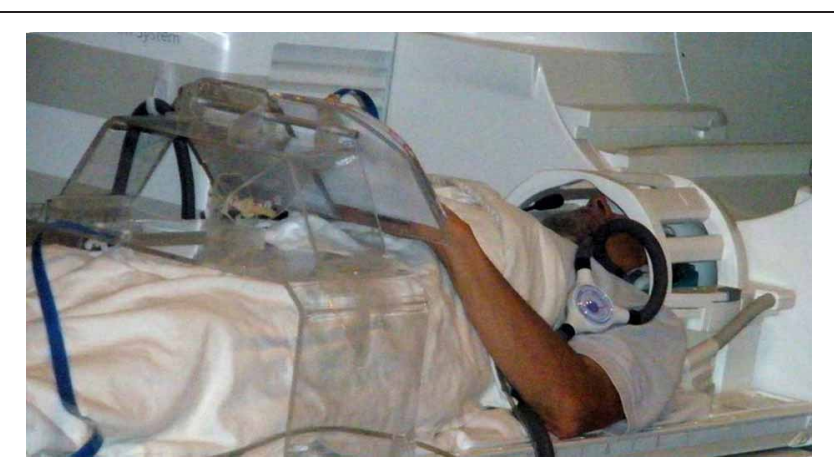

FIGURE 4 | A subject on the scanner bed operating our novel multimodal MRI-compatible drawing device. The plexiglass gantry supports a drawing tablet while a fiber-optic drawing stylus captures and records the drawing movements with high precision. The motion capture information synchronized with the fMRI allows the effect of behavioral events to be analyzed. movements. To our knowledge, this is the first multisensory drawing-system to support the fMRI investigation of tactilelyguided drawing by providing for the presentation of multiple tactile raised-line images in the scanner without the need of any operator. It also allows us to record relevant behavioral and feedback events and to correlate them to the brain activation for full off-line analysis.

\section{Auditory cue presentation}

The auditory stimuli were presented through Resonance Technologies Serene Sound earphones (Resonance Technologies, Salem, MA). To reduce scanner noise, this equipment employs external ear protectors with perforated ear plugs that conduct the auditory cues directly into the auditory passage while blocking much of the scanner noise.

\section{MRI DATA COLLECTION, ANALYSIS, AND VISUALIZATION fMRI acquisition}

MR data were collected on a Siemens Trio 3T scanner equipped with 8-channel EXCITE capability, a visual stimulus presentation system, response buttons. A high-resolution anatomical (T1weighted) volume scan of the entire brain was obtained for each observer $($ voxel size $=0.8 \times 0.8 \times 0.8 \mathrm{~mm})$. The fMRI bloodoxygenation-level-dependent (BOLD) responses were collected with EPI acquisition from the whole head coil. There were 34 axial slices at $2 \mathrm{~s} \mathrm{TR}$, with TE of $28 \mathrm{~ms}$ and flip angle of $80^{\circ}$, providing $3.0 \times 3.0 \times 3.5 \mathrm{~mm}$ voxels throughout the whole brain. The functional activations were processed for slice-time correction and motion correction. The two-phase motion correction consisted of a within-scan correction and a between-scan correction of each scan to the reference scan, both of which used mrVista (Stanford Vision and Imaging Science and Technology) to correct for six parameters of rigid-body motion. An anatomical segmentation algorithm (mrGray) was applied to the T1 scan, ensuring localization of the signal within the cortical gray matter close to the activated neurons and greatly reducing the blood drain artifacts that afflict studies in which cortical segmentation is not used. The activation was specified in terms of the statistical significance $(p<0.05)$ of the signal in each voxel (after Bonferroni correction for the number of gray matter voxels).

\section{Pre-processing}

The raw DICOM-format data from each fMRI scan were converted to a 4D NIFTI file. Using FSL tools, we ran within-scan and between-scan motion corrections, bringing all functional data into alignment with the fMRI volume acquired closest in time to the T1-weighted "inplane" anatomy. Then we averaged across scans, resulting in a single 4D NIFTI file for that scan session.

\section{fMRI time course analyses}

The data were analyzed to estimate the effective neural activation amplitudes (for each task across the 12 repeats of the 3-task sequence in a one-hour scan) by the following procedure. A General Linear Model (GLM) consisting of a $(3+1)$-parameter boxcar neural activation model convolved with an estimated hemodynamic response function (HRF) was fitted to the BOLD responses for each 3-task sequence, combined with a 1-parameter 
boxcar corresponding to the 8 auditory cue presentations and an additive 4 th-order polynomial to capture low-frequency drift in the BOLD signal. Thus, the parameters of the activation model consisted of the boxcar activation amplitudes for the three task periods, combined with the amplitudes of the auditory signals. (The HRF parameters were determined once per session by optimizing this model to a subset of gray matter voxels identified as most responsive to the task/rest alternation frequency in this experiment.)

\section{Voxel-wise parametric maps}

For each task-E/M, $M D$, and $S$-statistical parametric maps were generated, based on the estimated activation amplitudes from the above GLM in each voxel that exceeded the noise threshold defined by the variability across the 12 repeats of the 3-task sequence in each one-hour scan. Also, voxel-wise maps of the change in activation following the training period were generated, scaled in terms of $z$-score of the pre-post difference signals.

\section{ROI activation analysis}

The effective neural activation amplitudes (bar graphs) for each condition in each region of interest (ROI) were estimated by the same GLM procedure but now applied to the average signal across all voxels within the ROI. This procedure also provided highquality time courses for evaluation of the response dynamics and its comparison across tasks and stages of training.

The confidence intervals were defined by the amplitude variability the 12 repeats of the 3-task sequence in each one-hour scan. The dashed lines and the error bars represent confidence intervals for two different forms of statistical comparison of the activation levels (i.e., of the beta weights for the event types in the GLM): (1) The dashed lines represent the 99\% "zero" confidence interval ( $p<0.01$, uncorrected) within which the activation amplitudes are not significantly different from zero (i.e., relative to the noise variance for no stimulus-related activation defined as the residual variance after the GLM model fit of the FMRI time course analyses section described above); thus this statistical criterion is designed to indicate the significance of each individual activation (at $p<0.05$, corrected for multiple applications within each figure); (2) The error bars are "difference" confidence intervals designed to illustrate the $t$-test for the significance of differences between activation levels in each figure (i.e., the differences are not significant unless they exceed the confidence intervals for both compared activations), again at $p<0.05$ (corrected for multiple applications).

In the text, all ROI-comparisons are specified as significant by the $t$-test using a statistical criterion threshold of $p<0.05$ corrected for multiple comparisons.

\section{Topographic maps in the blind}

On the one hand, no informed analysis of the visual cortex could be done without knowledge of its retinotopic and functional organization; on the other hand, no retinotopic mapping or visual localizers are possible in the blind, so it was a challenge to localize any specific visual area. To resolve this issue and determine the borders of area V1 in blind participants, we took a three-pronged approach. First, we used the Freesurfer probability map atlas (see http://surfer.nmr.mgh.harvard.edu/fswiki/Brod
mannAreaMaps), to transform the primary visual area map back to the blind subject's brain through the Freesurfer spherical surface registration procedure. To verify the process, we first ran this procedure in the brains of sighted subjects, for which we already had individual retinotopic maps; the borders of the retinotopically defined V1 aligned fairly accurately with those from the Freesurfer. Second, we verified the location of the V1 ROI by intersecting with its anatomical marker (the calcarine sulcus). And third, we used an innovative 14-step procedure (Likova, 2010a,b, 2012) that allows us to warp the brains of sighted and blind subjects to the same MNI brain. This innovative threeway comparison enabled us to estimate the corresponding topographic regions in the blind brain. All methods converged very well to the definition of the V1 ROI.

\section{RESULTS}

\section{ENHANCED V1 ACTIVATION AFTER TRAINING TO DRAW FROM TACTILE MEMORY}

The focus of this analysis is the occipital region along the calcarine sulcus corresponding to the location of area V1. The V1 ROI was determined as explained in the Materials and Methods. Figure 5 shows a difference map for the MemoryDraw task, which represents voxel-wise comparison of the post-training BOLD activation relative to the pre-training level, projected on inflated representations of the medial views of the two hemispheres. It reveals strong post-training enhancement of the V1 activation (orange-yellow coloration within the green outlines) in both the left (LH) and the right $(\mathrm{RH})$ hemispheres.

\section{SUBJECT REPORT}

\section{Pre-training subject self-report}

Prior to training, subject CB4 reported a complete inability to comprehend the objects depicted by the raised-line models. All three tasks, even scribbling with a pen, were extremely challenging for her, and her performance was correspondingly poor. Although the familiarization session was sufficient to orient the subject to the experimental tasks and equipment, it did not advance her state beyond that of a total novice. Thus, a rudimentary functional organization was expected at this stage. In the first training session (after familiarization and the pre-training fMRI), CB4 spent an average of $183 \pm 43 \mathrm{~s}$ in completing each drawing.

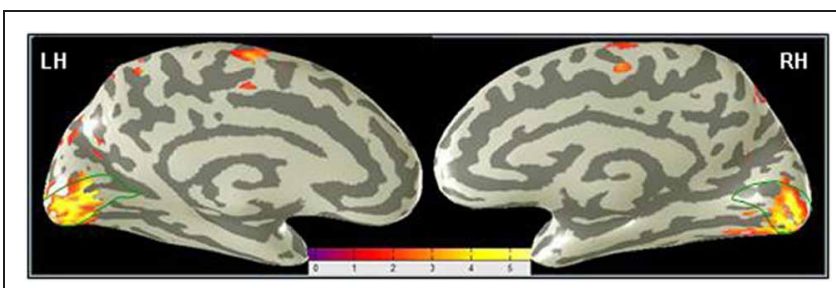

FIGURE 5 | Primary visual cortex shows the predominant learning effect in the $\boldsymbol{M D}$ task. A voxel-wise comparison, projected on inflated representations of the posterior left $(\mathrm{LH})$ and right $(\mathrm{RH})$ hemispheres, shows the increase (orange-yellow coloration) of the post-training BOLD activation in MD relative to the pre-training level. Dark gray, sulci; light gray, gyri. 


\section{Post-training subject self-report}

The post-training fMRI session was run eight weeks after the subject went through a week of 1-1.5 h/day training. Two "refresher" training sessions were also conducted in the week of the fMRI session. In the final training session, she spent an average of $23 \pm 3 \mathrm{~s}$ to complete each drawing, which is a highly significant improvement over the first training session $(p<0.0004$; $t=4.32 ; \mathrm{df}=19)$. The subject reported being able to recognize the raised-line drawings and generate a clear memory representation within the $20 \mathrm{~s}$ interval of the $E / M$ task; and $20 \mathrm{~s}$ later in the $M D$ task to recall the template from memory, to mentally "dissociate" it from the location where it was explored (the left slot of the tablet), to "project" it to the right-slot and to "trace" it there with the drawing stylus, as instructed during training. This report implies that robust memory representations, which are a prerequisite for guiding the complicated drawing movements, were successfully developed during the training period. Moreover, the dissociation from the initial location reflects successful learning of coordinate-transformation, which is known to be an important component of drawing, typically affected in some neurological conditions, such as constructional apraxia (e.g., Makuuchi et al., 2003; Ferber et al., 2007; Ogawa and Inui, 2009).

\section{COMPARATIVE PRE/POST-TRAINING ANALYSIS}

Comparison of the pre-training to post-training BOLD responses shows a dramatic enhancement from negligible activation in V1 before training (Figure 6A), to a massive task-specific activation as a result of training (Figure 6B).

\section{CROSS-TASK COMPARISON OF V1 ACTIVATION}

All cross-task ROI-comparisons in the text are specified as significant by the $t$-test using a statistical criterion threshold of $p<0.05$ corrected for multiple comparisons. The dashed lines and the error bars represent confidence intervals for two different forms

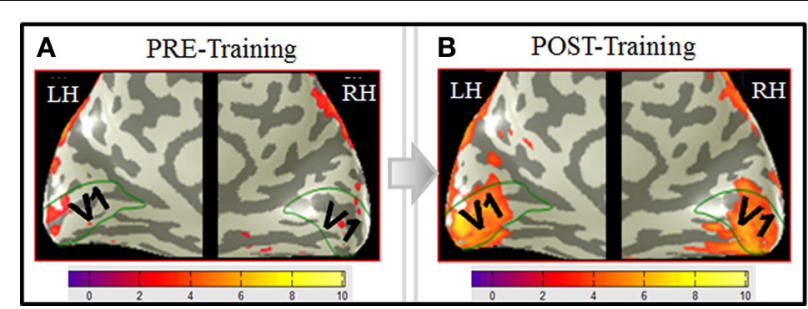

FIGURE 6 | $\mathrm{V} 1$ activation in $M D$ before training (A) and after training (B). BOLD activation (orange-yellowish coloration) from the MD task, derived according to the GLM described in Materials and Methods, and projected on inflated representations of the posterior left $(\mathrm{LH})$ and right $(\mathrm{RH})$ hemispheres is shown for both the pre-training $(\mathbf{A})$ and the post-training (B) fMRI sessions. Medial views of the posterior part of the brain optimally visualize area V1 (green outlines) along the calcarine sulcus. Scale bars show the color-coding for the $z$-score levels of the activation. Comparison of the pre- to post-training responses shows a dramatic enhancement from negligible activation in $\mathrm{V} 1$ before training (A), to a massive task-specific activation as a result of training (B). Note that, interestingly, the extension of the post-training activation approximately corresponds to the spatial extent of the images ( $10^{\circ}$ diameter) of statistical comparison of the activation levels (see "ROI activation analysis" section in "Materials and Methods" for more detail).

\section{Pre-training: lack of task-specificity}

Bar-graphs for the estimated activation in the V1 ROI in each hemisphere in (Figure 7A) indicate a lack of task-specificity (not significantly different activation levels, at $p<0.5$, corrected) for the $M D$ and both control tasks in the left hemisphere, with similar (NS at $p>0.5$ ) activation for $S$ in the right hemisphere, but noisy signals to $E / M$ and $M D$ in the right hemisphere.

\section{Post-training: memory task dominance}

Cross-task comparison of the V1 response for $M D$ (red bars) to those for $E / M$ (blue) and $S$ (green) in the left and right hemispheres after training are shown in Figure 7B. Note that, after training, the $M D$ response dominates in both left and right $\mathrm{V} 1$. As indicated by the confidence intervals, the following relationships are statistically significant ( $p<0.05$, corrected): $M D>$ $E / M$ and $M D>S$ in both hemispheres, and $M D>E / M>S$ in the left hemisphere. Thus, $M D$ was the task that most strongly activated V1 bilaterally, showing highly significant \% BOLD responses at low noise; the $E / M$ task gives significantly weaker, left-dominant responses; however, the motor-control scribbling task, $S$ (which lacks any memory component), is even suppressed in the left hemisphere.

\section{Pre/post comparison}

Comparison of the post-training response pattern to that before training (Figures 7A,B) shows the following statistically significant $\left(p<0.05\right.$, corrected) relationships: $E / M_{\text {post }} \cong E / M_{\text {pre }}$, $M D_{\text {post }}>M D_{\text {pre }}$ and $S_{\text {post }}<S_{\text {pre }}$ in both the left and the right hemispheres. This analysis implies a significant change in the V1 response pattern as a function of training. In particular, the V1 response in the memory-guided drawing task MD was substantially increased, while that of the non-memory motor control task $S$ was reduced effectively to zero.

\section{COMPARISON OF THE TIME-COURSE OF THE BOLD RESPONSE IN V1 Before training: immature $B O L D$ response waveforms in V1 (Figure 8A)}

Analysis of the time course of the BOLD responses underlying the estimated average response amplitude reveals deeper aspects of the neural processing at this initial stage of functional changes. As seen in Figure 8A, the average time courses for the sequence of the three task intervals (white bars) show substantial deviations of their waveforms (black lines) from the model prediction fits (color lines). The model takes into account both the task duration and the estimated HRF (see Materials and Methods). The pretraining response waveforms are rudimentary, poorly developed and noisy, with a prominent transient nature and early offsets long before the end of the $20 \mathrm{~s}$ task periods, in spite of the continuous hand movements during the full task period (as evident from the fully-fledged time course in the motor hand area, Figure 9A, and from the motion-capture records as well). These early offsets imply that the V1 neural response was essentially a brief transient pulse, suggesting an unsuccessful attempt to activate this area, 


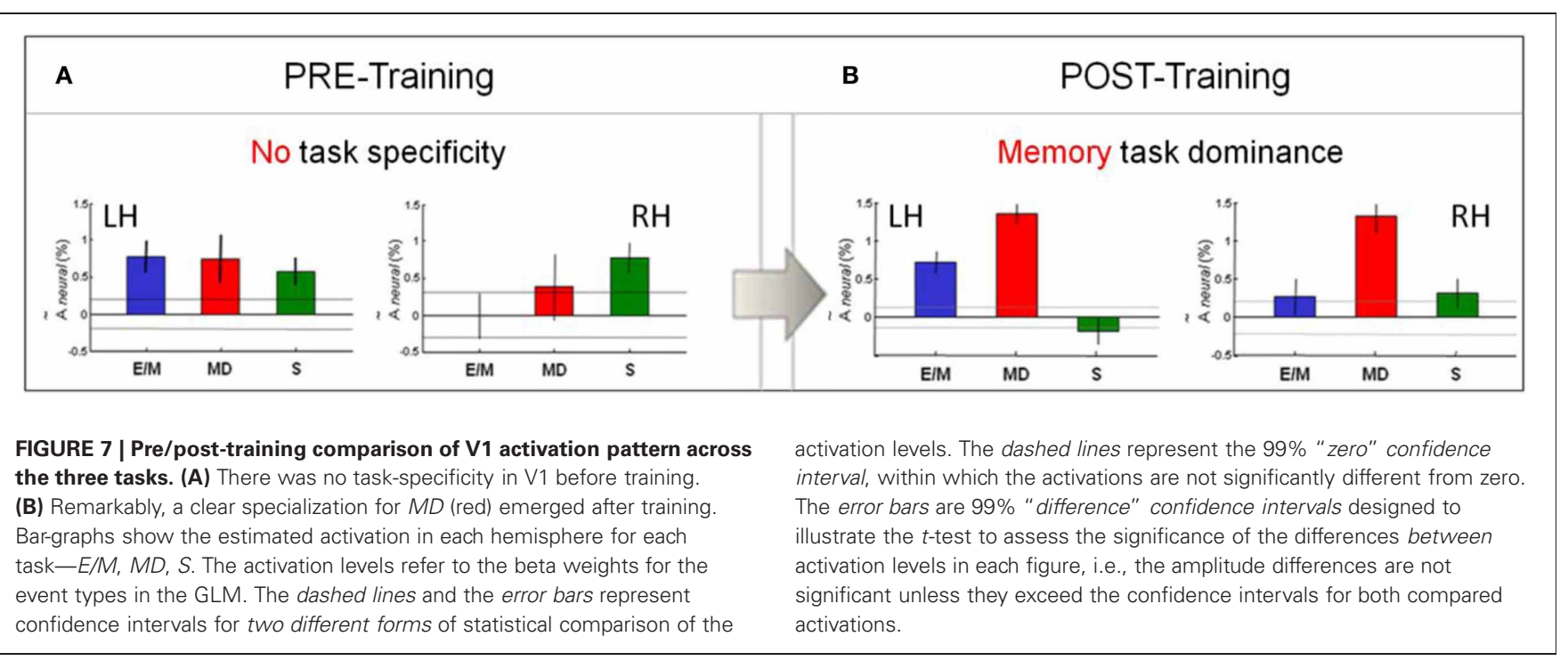

which was immediately withdrawn. Such undeveloped utilization of V1 is consistent with the subject self-report and drawing performance.

\section{After training: well-developed BOLD response waveform in V1 (Figure 8B)}

Notably, as a result of training, the V1 temporal waveforms became fully developed, i.e., a good match to the model prediction based on the sustained drawing activity throughout each task period (Figure 8B). We no longer see the transient earlyoffset signals. V1 responded very differently to the two types of drawing: while $M D$ generated the strongest signal bilaterally, the non-memory drawing $S$ was lacking any significant response.

\section{MOTOR CORTEX AS A CONTROL: MATURE SIGNALS, CLEAR TASK-SPECIFICITY \\ Before training: right hand specific, well-fitted BOLD response for both memory and non-memory drawing}

To verify that the rudimentary signals in V1 before training were not a general property of this brain, we also investigated the BOLD waveforms in non-deprived areas such as the hand area in the left motor cortex, which is well known to control right-hand movements. In contrast to V1, this area showed the expected functional specialization even before training: only the two right-hand tasks ( $M D$ and $S$, red and green bars, respectively) elicited activation, while the left-hand task $(E / M$, blue) did not (Figure 9A).

Furthermore, the signal waveforms were well-developed (black lines), conforming to the prediction of the neural activation model (color lines). These results thus verify that, despite the transient nature of the signals in V1, this congenitally blind cortex was able to generate normal responses in other areas before training.

\section{After training: BOLD response characteristics similar to the pre-training session}

Comparative analysis in the motor area shows that, as in the pretraining session (Figure 9A), the post-training signal waveforms were fully-developed and fitted by the model, with equally strong responses to $M D$ and $S$ (Figure 9B). These results indicate that

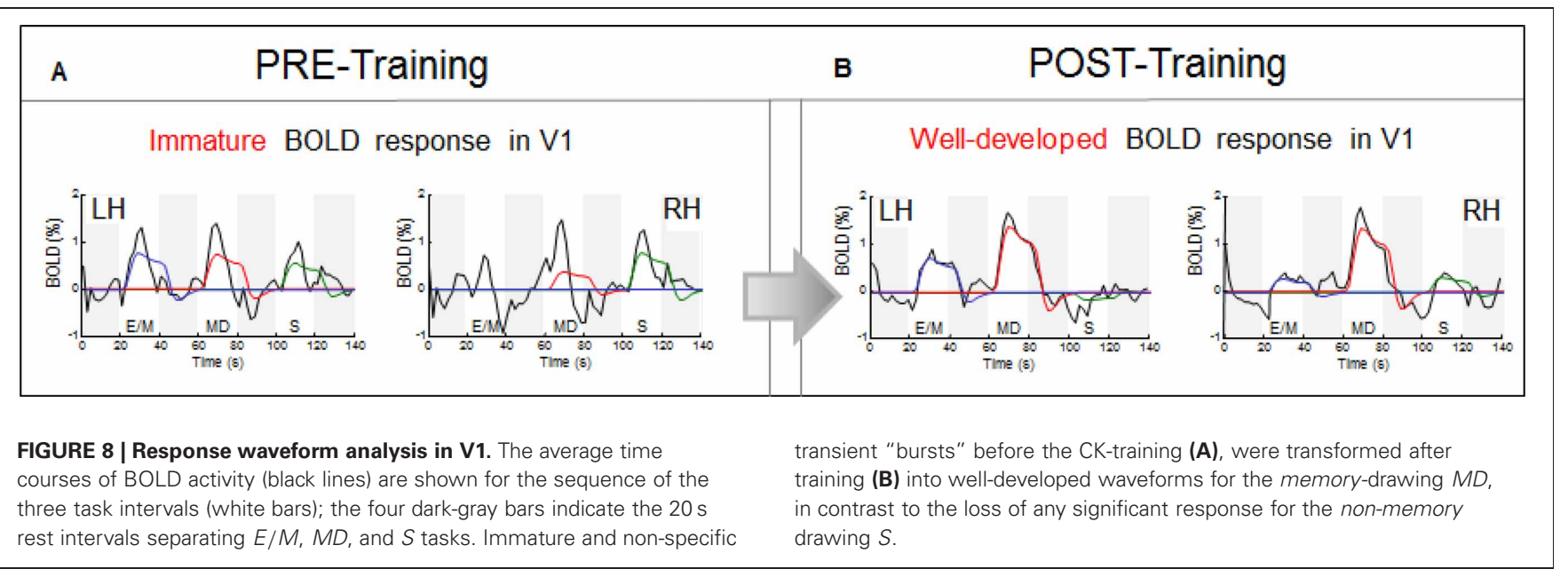




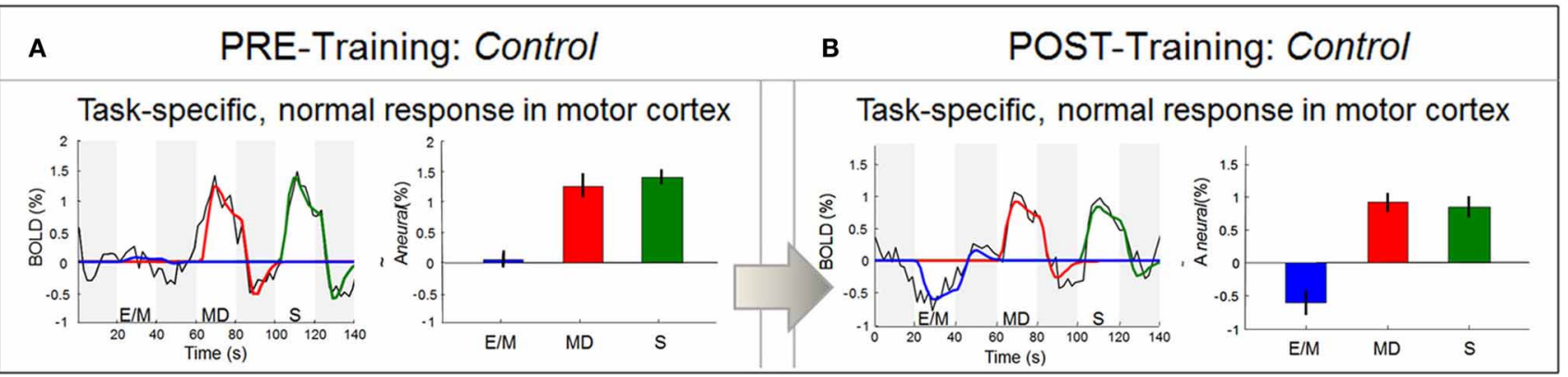

FIGURE 9 | Response waveform analysis in motor cortex. In contrast to area V1, the LH motor cortex M1 (in particular, the region encoding right-hand movement) produced well-formed response waveforms both before training (in $\mathbf{A}$ ) and after training (in $\mathbf{B}$ ). As should be expected, these responses were strong and positive for the two right-hand tasks (MD and $S$ ), and zero to negative for the left-hand task (E/M). Statistics as in Figure 7, and Materials and Methods. the hand motor area does not discriminate between the memoryand non-memory tasks, but treats them as two similar right-hand tasks, both before and after training. In contrast, after training $\mathrm{V} 1$ responded strongly to the memory task but not to the nonmemory task.

\section{DRAWING RESULTS}

Before training, the drawings of the congenitally blind subject were unrecognizable scrawls (Figure 10, middle panels), consistent with her self-report of an inability to comprehend the object depicted by the raised-line drawings. The training, however, was effective in developing CB4's capability to produce well recognizable drawings under non-visual memory guidance (Figure 10, right panels), consistent with her reporting of now being able to recognize and recall clearly detailed spatial representations.

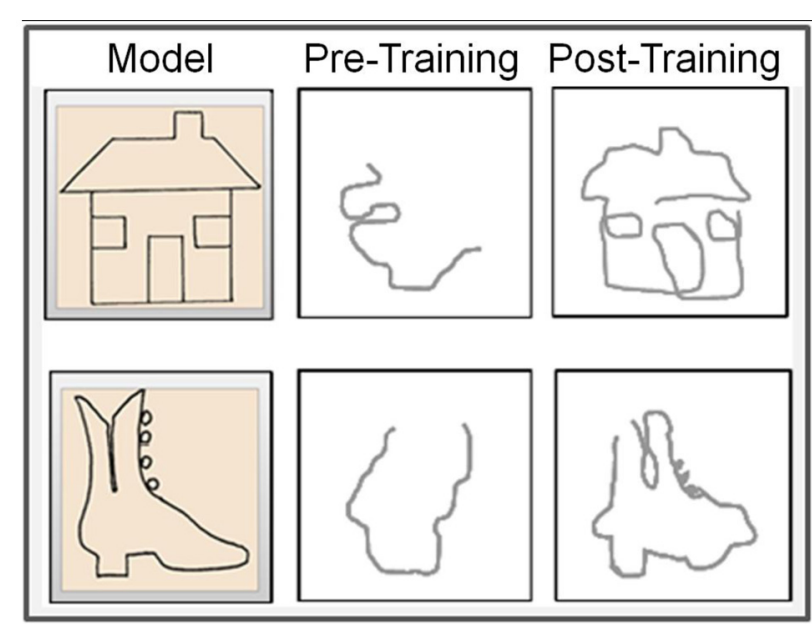

FIGURE 10 | Representative examples of pre- vs. post-training drawings. The left panels show the respective raised-line models to be drawn. Note the significant advance from practically unrecognizable drawings before training (middle panels) to well-recognizable drawings achieved by this totally blind subject as a result of the Cognitive-Kinesthetic training (right panels)

\section{DISCUSSION}

Training to draw in an absolute novice, who has been blind from birth, allowed us to investigate for the first time the evolution of the temporal dynamics of the functional reorganization in area $\mathrm{V} 1$, and the effect of learning to draw from tactile memory. Comparative pre/post-training analysis of the V1 BOLD response waveforms revealed their remarkable change from being transient and task non-specific (Figure 8A), to becoming full-fledged and task-specific (Figure 8B), and extending to a particular eccentricity in the cortical map (Figure 6B).

Except for her blindness and reduced hearing, this congenitally blind subject was in excellent physical and mental health, so there were no reasons to expect abnormal BOLD responses. However, it became evident that a reliance on tactile perception in her everyday life was insufficient to lead to development of the specific V1 functionality demanded by the $M D$ task, which showed undeveloped waveforms before training. Conversely, the well-developed response patterns in the hand motor area both before and after training (Figures 9A,B) confirmed that the abnormalities in V1 were a signature of its lack of relevant functional specialization before training, not an idiosyncratic subject response characteristic. It is important to stress that the use of the learning paradigm as an empirical intervention allowed us to go beyond mere task/activation correlation to the causal inference that the changes in V1 were a result of the training in the tactile-memory drawing task. Moreover, the concurrent measures of the objective memory readout (the drawings recorded by our motion-capture system) and the pre/post subject self-reports converged with fMRI evidence for the causal efficacy of the drawing training.

\section{MAIN PRINCIPLES}

This study is based on several basic principles. First, as emphasized by the capability of blind drawing, the "space" domain is not represented solely by vision: although the visual system is the modality best suited to process spatial information, it is not the only one; if deprived of visual input, the brain is capable of employing the "free" visual processing resources in the most relevant way. Second, learning an unusual, demanding task (such as drawing in blind adults) is a fruitful paradigm for studying brain reorganization and its developmental stages. Third, to provide for elaborated training in active spatial cognition, the training task 
has to encompass a large range of perception-to-action components (such as drawing). Finally, an effective way to force the learners to develop robust memory representations is to have a task that demands the explicit re-expression of these representations through the active motor loop (i.e., to "communicate back" these representations through drawing). Thus, the nonvisual drawing incorporating all these principles is a powerful experimental and memory training paradigm.

\section{COGNITIVE-KINESTHETIC TRAINING METHOD AND BLINDNESS}

Typically, studies in blind individuals are not done in a training paradigm, but rely on spontaneous experience-based plasticity and simply compare their current state with that of sighted individuals. The development of the Cognitive-Kinesthetic training method for non-visual drawing emphasizes a different approach. By implementing the above principles, this method presents a powerful research, and potentially, rehabilitation tool. By effectively teaching blind adults on a short time-scale, it allows the natural dissociation of cross-modal processing subsystems. Furthermore, it opens a window to observing the evolution of reorganization at both neural and behavioral level, and has the advantage of working with adults who can provide clear introspection and produce complex behavioral measures (as oppose to working with difficult-to-communicate infants).

Even the pre-training results are remarkable in capturing a very early developmental stage, one that is usually difficult to observe. The non-specific and immature pre-training responses seem to reflect the initial stage of functional "search," when the brain is still "probing" for the best resources before reaching the needed functional capability.

The self-reports of the congenitally blind subject before and after training were consistent with the changes observed in the V1 BOLD response (i.e., Figures 7A and $\mathbf{8 A}$, before training; and Figures $\mathbf{7 B}$ and $\mathbf{8 B}$, after training). Thus, the CognitiveKinesthetic training helped to maximize the ability for spatial reasoning and detailed memory representations with clear understanding of how the $2 \mathrm{D}$ tactile images being drawn relate to the depicted 3D objects.

\section{DRAWING AS A MEMORY PARADIGM}

The particular innovation of the neuroimaging experiments was to incorporate the drawing-based memory paradigm in the context of the Cognitive-Kinesthetic training method, together with the technological advances of the multisensory MRI-compatible system. This novel memory paradigm has the unique advantage of providing an explicit memory "readout" of the specific mental representation that guides it. Importantly, as in the case of the blindfolded study (Likova, 2010a, 2012), the CognitiveKinesthetic training enabled CB4 to draw from memory the specific memorized objects and faces that she had explored, not just some longstanding "clichés," thus showing that the particular memory-representations generated during the tactile exploration phase were guiding her drawing activity.

\section{RELATION TO PREVIOUS STUDIES}

In general, our results are consistent with previous reports in showing that brain areas traditionally considered purely visual, such as the primary visual area V1, can be activated in a crossmodal manner. Braille reading, naming, auditory localization, tactile discrimination, and other non-visual perception tasks can lead in the blind to reorganization and recruitment of visual cortex in a compensatory manner (e.g., Uhl et al., 1991, 1993, Sadato et al., 1996; Cohen et al., 1997; deVolder et al., 1997; Buechel et al., 1998; Hamilton et al., 2000; Burton et al., 2002; Amedi et al., 2003, 2004, 2008; Gizewski et al., 2003; Theoret et al., 2004; Merabet et al., 2005; Pascual-Leone et al., 2005; Voss et al., 2006; Goyal et al., 2006; Borowsky et al., 2007; Ptito et al., 2008; Amedi et al., 2008).

The present study extends these results to tactile memory and the effect of training. We asked if area $\mathrm{V} 1$ is involved in tactile working memory in a congenitally blind individual who was a total novice in a highly demanding tactile-memory task. The rapid recruitment of adult primary "visual" cortex as a result of a shorttime Cognitive-Kinesthetic training implies the operation of a much faster form of learning-based plasticity than exhibited by the many decades of self-training in the blind artist Armagan (Amedi et al., 2008).

As the post-training activation in V1 in the memory drawing task was generated without either visual or tactile sensory stimulation, this paradigm excludes explanations based not only on visual bottom-up input, but also on direct signals between primary sensory cortices.

\section{INTERPRETATION OF V1 AS A MEMORY BUFFER}

To put the present results in specific perspective, we needed concepts relevant to the mechanisms involved in the memory encoding and retrieval for the kinds of spatial structures used in the drawing task. The spatial memory buffer construct logically provides one likely framework. The MemoryDraw task is an active task that demands not only a physical sketchpad, but an internal "sketchpad" on which to "project," hold and manipulate the memory representation, so as to be able to use it to guide the trajectory of the complicated drawing movements. These demands closely resemble (although in a non-visual form) the description of the visuo-spatial memory buffer, termed the "visuo-spatial sketchpad." In the classic model of working memory (as proposed by Baddeley and Hitch, 1974; Baddeley, 1986, 2000, 2003), the visuo-spatial sketchpad is one of four major components, the one that instantiates the function of developing and holding in working memory an accurate spatial representation of the retrieved object to guide action, providing a "sketch" that can be further spatially manipulated by the central executive (Figure 11). The improvement of drawing performance we observed would not have been possible without recourse to a working memory representation of this kind.

An intriguing issue, of course, is where in the brain such representational buffer may be located. Previous theoretical and neurophysiological studies have proposed that area V1, is the cortical region best suited to perform such memory-buffer function (e.g., Mumford, 1996; Lee et al., 1998; Super et al., 2001a,b; Lee and Mumford, 2003; Super, 2003). This area has the special status of being the largest topographic map in the brain, with the highest spatial resolution and parallel processing of the information of the whole map; thus it has been suggested that "instead of being 


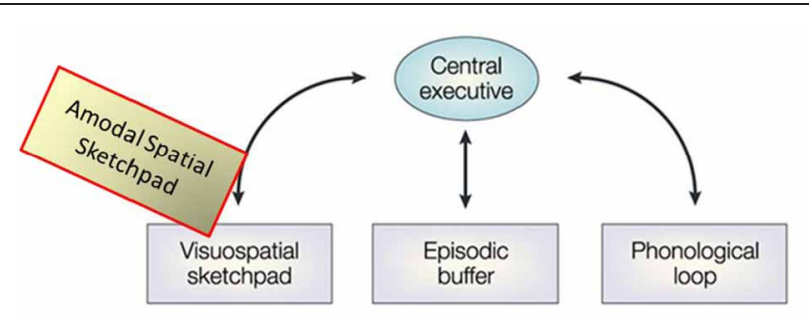

FIGURE 11 | Re-conceptualization of the visuo-spatial sketchpad as an amodal-spatial sketchpad. Modified schematic of the main modules of Baddeley's classic model of working memory including the visuo-spatial sketchpad [after Baddeley, 2003], where the added "Amodal-Spatial

Sketchpad" block depicts our re-conceptualization of the visuo-spatial sketchpad as being accessible to any sensory modality.

the first stage in a feedforward pipeline, V1 is better described as the unique high-resolution buffer in the visual system" (Lee and Mumford, 2003).

The current study provides a causal manipulation that links the memory enhancement to the increased activation and specialization in V1, consistent with the memory-buffer interpretation. Moreover, an important twist for this interpretation is the lack of any visual stimulation in the congenitally blind case (as it was in the blindfolded study), implying that the buffer is independent of the input modality. Our re-conceptualization of the visuo-spatial sketchpad as being amodal-spatial is depicted by the yellowish block in Figure 11. Indeed, the original motivation for the study was our view that, although vision provides the best spatial representation, space itself is not "owned" by vision, but is inherently an amodal domain. Thus, it is adaptively effective for the brain to ensure the modality-independence of spatial representation.

\section{ALTERNATIVE INTERPRETATIONS}

An alternative interpretation of the V1 activation to be evaluated in this congenitally blind case is its possible role in visual imagery. The interrelationships between working memory and imagery are still unclear and challenging. Although each of these major cognitive constructs is treated in various ways across studies, often without any attempt at formal definition, they all accept that both imagery and working memory involve a type of internal representation available to our awareness. In working memory, however, there is a further emphasis on goal-oriented, active maintenance, and use of this conscious representation to guide voluntary action; for this purpose, the multicomponent working memory models incorporate representational buffers, such as the visuo-spatial sketchpad discussed above, plus central executive functions (Figure 11).

In general, any form of retrievable and robust spatial memory "sketch," including one involving visual imagery, might in principle provide a mechanism for guiding the drawing movements. On the other hand, it has to be taken into account that the representational format and even the very nature of imagery are not yet resolved and are still a subject of hot debates even in the visual domain. Thus, "the analog-propositional debate, occasionally also called the picture-description debate, is an ongoing and notoriously irreconcilable dispute within cognitive science about the representational format of visual imagery," Stanford Encyclopedia of Philosophy (http://plato.stanford.edu/ entries/mental-imagery/).

In contrast to late-onset blind individuals, who (similarly to the sighted) have had enough visual stimulation to develop vision and visual imagery, congenitally blind individuals have had no access to visual information. Thus, it is accepted as "clearly true that visual imagery does not account for cross-modal activation of visual cortex for the early blind" (Lacey et al., 2009), and that congenitally blind are unable to perform visual imagery tasks (e.g., Goyal et al., 2006). Besides, it is logically impossible to ascertain whether the congenitally blind have visual imagery, as they have no previous vision-related experience and hence no basis for such a subjective qualia comparison.

Besides, an objective property of visual imagery is that its underlying cortical signals propagate in a top-down fashion through the occipital visual hierarchy, with the signal being strongest in the higher extrastriate areas (e.g., Ishai and Sagi, 1995; Kreiman et al., 2000; O'Craven and Kanwisher, 2000; Kosslyn et al., 2001; Tong, 2003; Mechelli et al., 2004; Amedi et al., 2005; Merabet et al., 2005), and possibly not reaching V1 at all; thus, there is still an open debate whether imagery activates V1 itself or not. The above characteristics of the imagery response in the visual hierarchy, and in V1 in particular, are highly incompatible with the strong $\mathrm{V} 1$ activation in CB4 under our tactile-memory task; while, in contrast, this strong and mature activation following the memory training is more consistent with the alternative hypothesis of $\mathrm{V} 1$ operating as a memory buffer.

\section{WHAT NEURAL MECHANISMS MAY BE ACTIVATING V1?}

The novel memory paradigm, based on training in drawing guided solely by tactile memory, opens an intriguing domain for future research. If the putative spatial buffer or "sketchpad" for working memory is implemented in V1, does imagery involve the same passive buffer? Alternatively, does working memory employ an imagery-specific representational mechanism to occupy our awareness; or do they both utilize a more generic "projection screen" (of either modality-specific or modality-independent nature)? All of these important questions are still open.

In particular, further studies in a wider population are needed to investigate what deeper mechanisms may mediate the crossmodal reorganization observed in $\mathrm{V} 1$. There is a range of theoretical possibilities, such as unmasking of preexisting connections, changes in synaptic weights, growth of new connections, modulation of long-range influences, up-regulation of specific transmitter sources, or a combination of a number of different mechanisms (e.g., Florence and Kaas, 1995; Jones, 2000; Raineteau and Schwab, 2001; van Brussel et al., 2011; Merabet et al., 2008b). Indeed, the extent of V1 connectivity is currently undergoing an extensive re-evaluation. Recent electrophysiological and anatomical studies in non-human primates reveal a picture of multiple reciprocal connections at lower hierarchical levels, including the primary areas. An impressive number of interconnections to and from V1 across the brain have been established. In addition to the well-known direct feedback projections to V1 originating from V2, V3, V4, V5 or MT, MST, FEF, LIP, and inferotemporal 
cortex (Perkel et al., 1986; Ungerleider and Desimone, 1986a,b; Shipp and Zeki, 1989; Rockland, 1994; Budd, 1998; Barone et al., 2000; Suzuki et al., 2000), there are direct feedforward projections to V1 originating from the pulvinar, LGNd, claustrum, nucleus paracentralis, raphe system, locus coeruleus, and the nucleus basalis of Meynert (Ogren and Hendrickson, 1976; Rezak and Benevento, 1979; Graham, 1982; Blasdel and Lund, 1983; Doty, 1983; Perkel et al., 1986; Lachica and Casagrande, 1992; Hendry and Yoshioka, 1994; Adams et al., 2000; Schmolesky, 2000).

Other potential candidate sources of direct V1 activation'were considered by Merabet et al. (2007), such as long-range corticocortical connections from multimodal parietal areas (Rockland and Ojima, 2003), or from somatosensory or other primary sensory cortices (Falchier et al., 2002; Cappe and Barone, 2005), plastic changes in subcortical pathways (e.g., Sur and Leamey, 2001), or multisensory interactions within primary sensory areas mediated by a competing balance between a form of direct drive and potentially inhibitory top-down projections from associative cortical areas. Even so, the connections listed above still represent only a subset of the direct and indirect projections that carry signals into and out of $\mathrm{V} 1$. That interconnectivity provides a number of potential sources of direct V1 activation, one of which is the pulvinar, since the anatomical connectivity with the pulvinar is particularly strong.

Additionally, Clavagnier et al. (2004), examined feedback projections to area V1 using retrograde tracer injections. Notably, in addition to well-known areas and a number of long-distance feedback connections originating from auditory (A1) and multisensory (STP) cortices, they also found connections from a perirhinal area. The perirhinal-to-V1 connections are of particular interest in the context of our finding of a memory related role for V1, as they could represent another potential pathway for the involvement of $\mathrm{V} 1$ in working memory and the active processing of stored spatial information (or what Clavagnier et al. refer to as "consciousness"). Definitive studies on these issues remain to be conducted, however.

\section{IMPLICATIONS FOR BRAIN ORGANIZATION AND PLASTICITY}

The present results in this case of congenital blindness address a series of cogent questions in relation to cortical plasticity. What happens to the "territory" of the visual cortex once the visual input is lost? For example, it is well-known that the two eyes normally "cooperate" to provide our binocular vision capabilities. However, if the inputs to the two eyes become unequal for some reason, cooperation turns into competition. Such binocular competition often alters the cortical organization and results in pathological conditions such as amblyopia. An analogous competition is also operating at the subsequent hierarchical level-that of cross-modal interactions. When the diverse sensory inputs are in balance, the cooperative principle determines their integrative work. If this balance is seriously disturbed, as in blindness, then the stronger sensory modality has the capability of invading the visual territory through the compensatory cross-modal mechanisms of brain plasticity and reorganization. Deterioration or loss of vision thus induces cortical changes and corresponding shifts in the cortical weighting of information processing in order to adapt to both the modified infrastructure of available sensory inputs and the new demands to the brain (e.g., Pascual-Leone et al., 2005; Merabet et al., 2005; Merabet et al., 2008a; Borowsky et al., 2007; Ptito et al., 2008; Likova, 2012). The present results in CB4 imply that, therefore, this is not a "random walk incursion," but is ruled by sophisticated mechanisms accounting for the task-specific demands.

In terms of the time-course analysis, our finding of immature BOLD waveforms before training indicates that simply measuring some activation level is not a sufficient indicator of functional reorganization, and thus it has an important implication as a warning for neuroimaging studies on plasticity, implying that additional criteria, incorporating the characteristics of the response time-course, also have to be taken into account.

\section{CONCLUSION}

The present results have multivalent implications. Although there have been a lot of studies on cross-modal perception in the blind, there have been only a few studies on cross-modal memory, and even fewer have incorporated a training process. Thus, to our knowledge, the current study provides the first to assess the involvement of area V1 in a tactile-memory task, as well as being the first on the effect of training on both tactile-memory under visual deprivation and on learning to draw in congenital blindness. In our task, the tactile model under the fingers of the left hand is removed after being tactually explored and memorized, so the drawing movements of the right hand are guided solely by memory, with no concurrent tactile input from the model.

The brain of this congenitally blind adult showed rapid changes in the process of learning to draw. This issue is particularly telling in relation to V1, which was massively activated as the skill of drawing from tactile memory was enhanced. It may seem particularly surprising to find such reorganization in $\mathrm{V} 1$, whose main role is considered to be the early processing of information from the visual input modality. The implication is that, even late in life, CB4's visual cortex still retained enough plasticity to be selectively accessible for use when the need arose, such as in our demanding memory task. This novel experimental approach, accessing the highest resolution topographic map in the brain (V1), provides a "real-life" yet tractable paradigm for re-evaluating principles of brain architecture. Moreover, the Cognitive-Kinesthetic training method should contribute to effective rehabilitative interventions in the lives of those with sensory disabilities. It is particularly noteworthy that the training was transformative for the congenitally blind individual. Helmholtz' concept of drawing as empowering "particularly vivid and accurate" registration of sensory structures, their storage, and retrieval so as to provide true memory in a form that can be translated to guide the accurate motor-control signals, seems to be true even under blindness, as in the case of CB4.

The present findings, to the extent that they can be generalized, add a compelling slate of evidence against the view of exclusively sensory and unimodal primary cortices, thus being consistent with the idea of a more distributed architecture and increased "task sharing" among sensory processing regions even 
within the sensory neocortex. In combination with our previous work with the drawing paradigm, these studies propel the emerging re-conceptualization of brain architecture as highly interactive and capable of reorganization even after long-term sensory deprivation.

\section{REFERENCES}

Adams, M. M., Hof, P. R., Gattass, R., Webster, M. J., and Ungerleider, L. G. (2000). Visual cortical projections and chemoarchitecture of macaque monkey pulvinar J. Comp. Neurol. 419, 377-393.

Amedi, A., Floel, A., Knecht, S., Zohary, E., and Cohen, L. G. (2004). Transcranial magnetic stimulation of the occipital pole interferes with verbal processing in blind subjects. Nat. Neurosci. 7, 1266-1270.

Amedi, A., Malach, R., and PascualLeone, A. (2005). Negative BOLD differentiates visual imagery and perception. Neuron 48, 859-872.

Amedi, A., Merabet, L. B., Camprodon, J., Bermpohl, F., Fox, S., Ronen, I., Kim, D. S., and Pascual-Leone, A. (2008). Neural and behavioral correlates of drawing in an early blind painter: a case study. Brain Res. 1242, 252-262.

Amedi, A., Raz, N., Pianka, P., Malach, R., and Zohary, E. (2003). Early "visual" cortex activation correlates with superior verbal memory performance in the blind. Nat. Neurosci. 6, 758-766.

Baddeley, A. D. (1986). Working Memory. New York, NY: Oxford University Press.

Baddeley, A. D. (2000). The episodic buffer: a new component of working memory? Trends Cogn. Sci. 4, 417-423.

Baddeley, A. D. (2003). Working memory: looking back and looking forward. Nat. Rev. Neurosci. 4, 829-839.

Baddeley, A. D., and Hitch, G. J. (1974). "Working memory," in The Psychology of Learning and Motivation, Vol. 8, ed G. Bower (San Diego, CA: Academic Press), 47-90.

Barone, P., Batardiere, A., Knoblauch, K., and Kennedy, H. (2000). Laminar distribution of neurons in extrastriate areas projecting to visual areas V1 and V4 correlates with the hierarchical rank and indicates the operation of a distance rule. J. Neurosci. 20, 3263-3281.

Borowsky, R., Esopenko, C., Cummine, J., and Sarty, G. E. (2007). Neural representations of visual words and objects: a functional MRI study on the modularity of reading and object processing. Brain Topogr. 20, 89-96.

Blasdel, G. G., and Lund, J. S. (1983). Termination of afferent axons in macaque striate cortex. J. Neurosci. 3, 1389-1413.

Buchsbaum, B. R., and D'Esposito, M. (2009). "Is there anything special about working memory?" in Neuroimaging of Human Memory, eds F. Rosler, C. Ranganath, B. Roder, and R. Kluwe (Oxford: Oxford University Press), 255-265.

Budd, M. L. (1998). Extrastriate feedback to primary visual cortex in primates: a quantitative analysis of connectivity. Proc. R. Soc. Lond. B Biol. Sci. 265, 1037-1044.

Buechel, C., Price, C., Frackowiak, R. S., and Friston, K. (1998). Different activation patterns in the visual cortex of late and congenitally blind subjects. Brain 121, 409-419.

Burton, H., Snyder, A. Z., Conturo, T. E., Akbudak, E., Ollinger, J. M., and Raichle, M. E. (2002). Adaptive changes in early and late blind: a fMRI stud of Braille reading. J. Neurophysiol. 87, 589-607.

Cappe, C., and Barone, P. (2005). Heteromodal connections supporting multisensory integration at low levels of cortical processing in the monkey. Eur. J. Neurosci. 22, 2886-2902.

Clavagnier, S., Falchier, A., and Kennedy, H. (2004). Long-distance feedback projections to area V1: implications for multisensory integration, spatial awareness, and visual consciousness. Cogn. Affect. Behav. Neurosci. 4, 117-126.

Cohen, L. G., Celnik, P., PascualLeone, A., Corwell, B., Falz, L., and Dambrosia, J. (1997). Functional relevance of cross-modal plasticity in blind humans. Nature 389, 180-183.

de Renzi, E. (1982). Disorders of Space Exploration and Cognition. New York, NY: Wiley and Sons.

deVolder, A. G., Bol, A., Blin, J., Robert, A., Arno, P., and Grandin, C. (1997). Brain energy metabolism in early blind subjects: neural activity in the visual cortex. Brain Res. 750, 235-244.

Doty, R. W. (1983). Nongeniculate afferents to striate cortex in

\section{ACKNOWLEDGMENTS}

This research is supported by NSF/SLC Grant to Lora T. Likova. The author thanks Spero Nicholas for his help in data preprocessing and analysis tools, and Christopher W. Tyler for helpful discussions.

macaques. J. Comp. Neurol. 218, 159-173.

Falchier, A., Clavagnier, S., Barone, P., and Kennedy, H. (2002). Anatomical evidence of multimodal integration in primate striate cortex. J. Neurosci. 22, 5749-5759.

Ferber, S., Mraz, R., Baker, N., and Graham, S. J. (2007). Shared and differential neural substrates of copying versus drawing: a functional magnetic resonance imaging study. Neuroreport 18, 1089-1093.

Florence, S. L., and Kaas, J. H. (1995). Large-scale reorganization at multiple levels of the somatosensory pathway follows therapeutic amputation of the hand in monkeys. J. Neurosci. 15, 8083-8095.

Gardner, H. (1983). Frames of Mind: The Theory of Multiple Intelligences. New York, NY: Basic Books.

Gizewski, E. R., Gasser, T., de Greiff, A., Boehm, A., and Forsting, $\mathrm{M}$. (2003). Cross-modal plasticity for sensory and motor activation patterns in blind subjects. Neuroimage 19, 968-975.

Goyal, M. S., Hansen, P. J., and Blakemore, C. B. (2006). Tactile perception recruits functionally related visual areas in the late-blind. Neuroreport 17, 1381-1384.

Graham, J. (1982). Some topographical connections of the striate cortex with subcortical structures in Macaca fascicularis. Exp. Brain Res. 47, 1-14.

Grossi, D., and Trojano, L. (1999). "Constructional apraxia," in Handbook of Clinical and Experimental Neuropsychology, eds G. Denes and L. Pizzamiglio (Hove: Psychology Press), 441-450.

Hamilton, R., Keenan, J. P., Catala, M., and Pascual-Leone, A. (2000). Alexia for Braille following bilateral occipital stroke in an early blind woman. Neuroreport 11, 237-240.

Harrison, S. A., and Tong, F. (2009). Decoding reveals the contents of visual working memory in early visual areas. Nature 458, 632-635.

Heller, M. A. (ed.). (2000). "Touch, representation and blindness," in Debates in Psychology Series, (New York, NY: Oxford University Press), 67-98.
Hendry, S. H., and Yoshioka, T. (1994). A neurochemically distinct third channel in the macaque dorsal lateral geniculate nucleus. Science 264, 575-577.

Ishai, A. (2009). "Retrieving pictures from long-term memory," in Neuroimaging of Human Memory, eds F. Rosler, C. Ranganath, B Roder, and R. Kluwe (New York, NY: Oxford University Press), 265-281.

Ishai, A., and Sagi, D. (1995). Common mechanisms of visual imagery and perception. Science 268, 1771-1974.

Jones, E. G. (2000). Plasticity and neuroplasticity. J. Hist. Neurosci. 9, 37-39.

Kennedy, J. (1993). Drawing and the Blind. New Haven, CT: Yale University Press.

Kennedy, J. M. (2000). "Recognizing outline pictures via touch: alignment theory," in Touch, Representation and Blindness, ed M. A. Heller (Oxford: Oxford University Press), 67-98.

Kennedy, J. M., and Igor, J. (2003). Haptics and projection: drawings by Tracy, a blind adult. Perception 32, 1059-1071.

Kennedy, J. M., and Juricevic, I. (2006). Foreshortening, convergence and drawings from a blind adult. Perception 35, 847-851.

Kosslyn, S. M., Ganis, G., and Thompson, W. L. (2001). Neural foundations of imagery. Nat. Rev. Neurosci. 2, 635-642.

Kosslyn, M. S., and Thompson, W. L. (2003). When is early visual cortex activated during visual mental imagery? Psychol. Bull. 129, 723-746.

Kreiman, G., Koch, C., and Freid, I. (2000). Category-specific visual responses of single neurons in the human media temporal cortex. Nat. Neurosci. 3, 946-953.

Lacey, S., Tal, N., Amedi, A., and Sathian, K. (2009). A putative model of multisensory object representation. Brain Topogr. 21, 269-274.

Lachica, E. A., and Casagrande, V. A. (1992). Direct W-like geniculate projections to the cytochromeoxidase (CO) blobs in primate visual cortex: axon morphology. $J$. Comp. Neurol. 319, 141-158.

Lee, B. H., Chin, J., Kang, S. J., Kim, E-J., Park, K. C., and Na, D. L. 
(2004). Mechanism of the closingin phenomenon in a figure copying task in Alzheimer's disease patients. Neurocase 10, 393-397.

Lee, T. S., and Mumford, D. (2003). Hierarchical Bayesian inference in the visual cortex. J. Opt. Soc. Am. A Opt. Image Sci. Vis. 20, 1434-1448.

Lee, T. S., Mumford, D., Romero, R., and Lamme, V. A. F. (1998). The role of the primary visual cortex in higher level vision. Vision Res. 38, 2429-2454.

Likova, L. T. (2010a). The primary visual cortex as a modalityindependent 'screen' for working memory. J. Vis. 10, 776.

Likova, L. T. (2010b). "Drawing in the blind and the sighted as a probe of cortical reorganization," in Human Vision and Electronic Imaging, eds B. E. Rogowitz and T. N. Pappas, Proc. SPIE, 7527, 752708-752714.

Likova, L. T., and Nicholas, S. N. (2010c). Early visual cortex as an amodal-memory "screen": evidence from fMRI in the blindfolded and the congenitally blind individuals. Society for Neuroscience Annual Meeting, San Diego, CA.

Likova, L. T. (2011). Dynamics of cross-modal plasticity in the human brain. Perception 40, 14.

Likova, L. T. (2012). "The spatiotopic 'visual' cortex of the blind," in Proceeding of the SPIE/HVEI, eds B. E. Rogowitz, T. N. Pappas, and H. de Ridder. 7529, 8291-8317.

Loomis, K. M., and Klatzky, R. (2008). "Functional equivalence of spatial representations from vision, touch, and hearing: relevance for sensory substitutions," in Touch, Representation and Blindness, ed M. A. Heller (New York, NY: Oxford University Press), 67-98.

Makuuchi, M., Kaminaga, T., and Sugishita, A. (2003). Both parietal lobes are involved in drawing: a functional MRI study and implications for constructional apraxia. Brain Res. Cogn. Brain Res. 16, 338-347.

Mayer-Gross, W. (1935). Some observations on apraxia. Proc. R. Soc. Med. 28, 1203-1212.

Mechelli, A., Price, C. J., Friston, K. J., and Ishai, A. (2004). Where bottom-up meets top-down: neuronal interactions during perception and imagery. Cereb. Cortex 14, 1256-1265.

Merabet, L., Rizzo, J., Amedi, A., Somers, D., and Pascual-Leone, A. (2005). What blindness can tell us about seeing again: merging neuroplasticity and neuroprostheses. Nat. Rev. Neurosci. 6, 71-77.
Merabet, L. B., Pitskel, N. B., Amedi, A., and Pascual-Leone, A. (2008a). "The plastic brain in blind individuals: the cause of disability and the opportunity for rehabilitation," in Blindness and Brain Plasticity in Navigation and Object Perception, eds J. J. Rieser, D. H. Ashmead, F F. Ebner, and A. L. Corn (London: Lawrence Erlbaum Associates), 23-43.

Merabet, L. B., Hamilton, R., Schlaug, G., Swisher, J. D., Kiriakopoulos, E. T., Pitskel, N. B., Kauffman, T., and Pascual-Leone, A. (2008b). Rapid and reversible recruitment of early visual cortex for touch. PLoS One 3:e3046. doi: 10.1371/journal.pone.0003046

Merabet, L. B., Swisher, J. D., McMains, S. A., Halko, M. A., Amedi, A., Pascual-Leone, A., and Somers, D. C. (2007). Combined activation and deactivation of visual cortex during tactile sensory processing. J. Neurophysiol. 97, 1633-1641.

Mumford, D. (1991). On the computational architecture of the neocortex I: the role of the thalamocortical loop. Biol. Cyber. 65, 135-145.

Mumford, D. (1996). "Commentary on banishing the homunculus by H. Barlow," in Perception as Bayesian Inference, eds D. C. Knill and W. Richards (Cambridge, UK: Cambridge University Press), 501-504.

O'Craven, K., and Kanwisher, N. (2000). Mental imagery of faces and places activates corresponding stimulus-specific brain regions. Nature 331, 68-70.

Ogawa, K., and Inui, T. (2009). The role of the posterior parietal cortex in drawing by copying. Neuropsychologia 47, 1013-1022.

Ogren, M., and Hendrickson, A. (1976). Pathways between striate cortex and subcortical regions in Macaca mulatta and Saimiri sciureus: evidence for a reciprocal pulvinar connection. Exp. Neurol. 53, 780-800.

Pascual-Leone, A., Amedi, A., Fregni, F., and Merabet, L. (2005). The plastic human brain. Annu. Rev. Neurosci. 28, 377-401.

Perkel, D. J., Bullier, J., and Kennedy, H. (1986). Topography of the afferent connectivity of area 17 of the macaque monkey: a double labeling study. J. Comp. Neurol. 253, 374-402.

Piercy, M., Hecaen, H., and Ajuriaguerra, J. (1960). Constructional apraxia associated with unilateral cerebral lesion-left and right-sided cases compared. Brain 83, 225-242.

Ponchillia, P. E. (2008). "Non-visual sports and arts: fertile substrates for the growth of knowledge about brain plasticity in people who are blind or have low vision," in Blindness and Brain Plasticity in Navigation and Object Perception, eds J. J. Rieser, D. H. Ashmead, F. F. Ebner, and A. L. Corn (London: Lawrence Erlbaum Associates), 283-313.

Ptito, M., Fumal, A., de Noordhout, A. M., Schoenen, J., Gjedde, A., and Kupers, R. (2008). TMS of the occipital cortex induces tactile sensations in the fingers of blind Braille readers. Exp. Brain Res. 184, 193-200.

Raineteau, O., and Schwab, M. E. (2001). Plasticity of motor systems after incomplete spinal cord injury. Nat. Rev. Neurosci. 2, 263-273.

Ranganath, H. (2009). "Interrelationships between working memory and long-term memory," in Neuroimaging of Human Memory, eds F. Rosler, C. Ranganath, B. Roder, R. Kluwe (Oxford: Oxford University Press), 227-255.

Raz, N., Amedi, A., and Zohary, E. (2005). V1 activation in congenitally blind V1 activation in congenitally blind humans is associated with episodic retrieval. Cereb. Cortex 15, 1459-1468.

Rezak, M., and Benevento, L. A. (1979). A comparison of the organization of the projections of the dorsal lateral geniculate nucleus, the inferior pulvinar and adjacent lateral pulvinar to primary visual cortex (area 17) in the macaque monkey. Brain Res. 167, 19-40.

Rockland, K. S. (1994). "The organization of feedback connections from areaV2 (18) to V1 (17)," in Cerebral Cortex, vol. 10: Primary Visual Cortex in Primates, eds A. Peters and K. S. Rockland (NewYork, NY: Plenum), 261-299.

Rockland, K. S., and Ojima, H. (2003). Multisensory convergence in calcarine visual areas in macaque monkey. Int. J. Psychophysiol. 50, 19-26.

Sadato, N., Pascual-Leone, A., Grafman, J., Ibanez, V., Deiber, M. P., Dold, G., and Hallett, M. (1996). Activation of the primary visual cortex by Braille reading in blind subjects. Nature 380 , 526-528.

Schmolesky, M. (2000). The primary visual cortex. Available from: http:// webvision.med.utah.edu/Visual Cortex.html
Shipp, S., and Zeki, S. M. (1989). The organization of connections between areas V5 and V1 in macaque monkey visual cortex. Eur. J. Neurosci. 1, 309-332.

Super, H. (2003). Working memory in the primary visual cortex. Arch. Neurol. 60, 809-812.

Super, H., Spekreijse, H., and Lamme, V. A. F. (2001a). A neural correlate of working memory in the monkey primary visual cortex. Science 293, 120-124.

Super, H., Spekreijse, H., and Lamme, V. A. F. (2001b). Two distinct models of sensory processing observed in monkey primary visual cortex (V1). Nat. Neurosci. 4, 304-310.

Sur, M., and Leamey, C. A. (2001). Development and plasticity of cortical areas and networks. Nat. Rev. Neurosci. 2, 251-262.

Suzuki, W., Saleem, K. S., and Tanaka, K. (2000). Divergent backward projections from the anterior part of the inferotemporal cortex (area TE) in the macaque. J. Comp. Neurol. 422, 206-228.

Theoret, H., Merabet, L., and PascualLeone, A. (2004). Behavioral and neuroplastic changes in the blind: evidence for functionally relevant cross-modal interactions. J. Physiol. Paris 98, 221-233.

Tong, F. (2003). Primary visual cortex and visual awareness. Nat. Rev. Neurosci. 3, 219-229.

Uhl, F., Franzen, P., Lindinger, G. Lang, W., and Deecke, L. (1991). On the functionality of the visually deprived occipital cortex in early blind persons. Neurosci. Lett. 124, 256-259.

Uhl, F., Franzen, P., Podreka, I., Steiner, M., and Deecke, L. (1993). Increased regional cerebral blood flow in inferior occipital cortex and cerebellum of early blind humans. Neurosci. Lett. 150, 162-164.

Ungerleider, L. G., and Desimone, R. (1986a). Projections to the superior temporal sulcus from the central and peripheral field representations of Vl and V2. J. Comp. Neurol. 248, 147-163.

Ungerleider, L. G., and Desimone, R. (1986b). Cortical connections of visual area MT in the macaque. J. Comp. Neurol. 248, 190-222.

van Brussel, L., Gerits, A., and Arckens, L. (2011). Evidence for crossmodal plasticity in adult mouse visual cortex following monocular enucleation. Cereb. Cortex 21, 2133-2146.

Voss, P., Gougoux, F., Lassonde, M., Zatorre, R. J., and Lepore, F. (2006). A positron emission 
tomography study during auditory localization by late-onset blind individuals. Neuroreport 17, 383-388.

Williams, M., Baker, C. I., Opde Beeck, H. P., Shim, W. M., Dang, S., Triantafyllou, C., and Kanwisher, N. (2008). Feedback of visual object information to foveal retinotopic cortex. Nat. Neurosci. 11, 1439-1445.

Conflict of Interest Statement: The author declares that the research was conducted in the absence of any commercial or financial relationships that could be construed as a potential conflict of interest.
Received: 14 April 2011; accepted: 22 February 2012; published online: 14 May 2012.

Citation: Likova LT (2012) Drawing enhances cross-modal memory plasticity in the human brain: a case study in a totally blind adult. Front. Hum. Neurosci. 6:44. doi: 10.3389/fnhum. 2012.00044
Copyright (C) 2012 Likova. This is an open-access article distributed under the terms of the Creative Commons Attribution Non Commercial License, which permits non-commercial use, distribution, and reproduction in other forums, provided the original authors and source are credited. 